\title{
Caracterização do modelo de atenção básica à saúde bucal na região nordeste no período de 2015-2017
}

Characterization of the primary care model in oral health in northeast region in the period 2015-2017

Caracterización del modelo de atención básica a la salud bucal en la región nordeste en el período de 2015-2017

Samara Ellen da SILVA 1

João Horácio Pereira de ARAÚJO ${ }^{1}$

Isla Camilla Carvalho LAUREANO

Lunna FARIAS ${ }^{2}$

Catarina Ribeiro Barros de ALENCAR ${ }^{3}$

Alessandro Leite CAVALCANTI ${ }^{4}$

'Graduando(a) em Odontologia Universidade Estadual da Paraíba, UEPB, 58429-500 Campina Grande-PB, Brasil

${ }^{2}$ Mestranda em Odontologia pelo Programa de Pós-Graduação em Odontologia, Universidade Estadual da Paraíba, UEPB, 58429-500 Campina Grande-PB, Brasil

${ }^{3}$ Pós-Doutoranda do Programa de Pós-Graduação em Odontologia, Universidade Estadual da Paraíba UEPB, 58429-500 Campina Grande-PB, Brasil

${ }^{4}$ Professor Doutor do Programa de Pós-Graduação em Odontologia da Universidade Estadual da Paraíba UEPB, 58429-500 Campina Grande-PB, Brasil

\section{Resumo}

Introdução: Os Sistemas de informação têm como finalidade promover a elaboração e facilitar a avaliação de políticas, planos e programas de saúde, uma vez que seus indicadores refletem a saúde da população. Objetivo: O presente estudo tem como objetivo caracterizar o modelo de atenção básica à saúde bucal na região Nordeste do Brasil, a partir de dados secundários da produção ambulatorial em saúde bucal, disponíveis no SIA-SUS. Material e método: Tratou-se de estudo descritivo e quantitativo, utilizando dados secundários a partir da produção ambulatorial da atenção básica em saúde bucal, disponíveis no Sistema de Informações Ambulatoriais do Sistema Único de Saúde (SIASUS). A coleta dos dados foi feita a partir do banco de dados do Departamento de Informática do SUS (DATASUS) e considerou o período de janeiro de 2015 a dezembro de 2017. Foram incluídos no estudo os dados sobre os procedimentos curativos e preventivos realizados em ambiente ambulatorial pelos cirurgiões-dentistas. Os dados foram apresentados por meio de estatística descritiva. Resultados: Obteve-se em 2015, o maior número total de procedimentos realizados (52.801.784). Do total de procedimentos curativos entre 2015-2017, tem-se maioria de procedimentos restauradores $(22,7 \%)$ e minoria de procedimentos cirúrgicos $(15,2 \%)$. Observa-se que, nos três anos estudados, a produção de procedimentos curativos superou a de procedimentos preventivos. Conclusão: O modelo básico de atenção à saúde bucal no Nordeste apresentou caráter curativo, com predominância dos procedimentos restauradores, seguidos pelos periodontais, ficando em menor número os procedimentos cirúrgicos.

Descritores: Saúde Pública; Sistemas de Informação em Atendimento Ambulatorial; Saúde Bucal.

\section{Abstract}

Introduction: Information systems aim to promote the elaboration and facilitate the evaluation of policies, health plans and programs, since their indicators reflect the health of the population. Objective: The objective of this study is to characterize the primary care model for oral health in the Northeast region of Brazil, based on secondary data on ambulatory oral health production available at SIA-SUS. Material and method: This was a descriptive and quantitative study, using secondary data from the outpatient production of primary care in oral health, available in the Outpatient Information System of the Unified Health System (SIA-SUS). Data collection was done from the database of the Department of Information Technology of the SUS (DATASUS) and considered the period from January 2015 to December 2017. Data were included in the study on curative and preventive procedures performed in an outpatient setting by dentists. The data were presented through descriptive statistics. Results: The highest number of procedures performed $(52,801,784)$ was obtained in 2015 . Of the total curative procedures between 2015-2017, a majority of restorative procedures (22.7\%) and minority of surgical procedures (15.2\%) were performed. It is observed that in the three years studied, the production of curative procedures surpassed that of preventive procedures. Conclusion: The the primary care model for oral health in the Northeast was curative, predominantly restorative procedures, followed by periodontal procedures, with fewer surgical procedures.

Descriptors: Public Health; Ambulatory Care Information Systems; Oral Health.

\section{Resumen}

Introducción: Los sistemas de información tienen como finalidad promover la elaboración y facilitar la evaluación de políticas, planes y programas de salud, ya que sus indicadores reflejan la salud de la población. Objetivo: El presente estudio tiene como objetivo caracterizar el modelo de atención básica a la salud bucal en la región Nordeste de Brasil, a partir de datos secundarios de la producción ambulatoria en salud bucal, disponibles en el SIA-SUS. Métodos: Se realizó un estudio descriptivo y cuantitativo, con datos secundarios obtenidos a partir del tratamiento ambulatorio de atención dental básica disponible en el Sistema de Información Ambulatoria del Sistema Único de Salud (SUS-SIA). La recolección de los datos fue hecha a partir del banco de datos del Departamento de Informática del SUS (DATASUS) y consideró el período de enero de 2015 a diciembre de 2017. Se incluyeron en el estudio los datos sobre los procedimientos curativos y preventivos realizados en ambiente ambulatorio por los cirujanos-dentistas. Los datos fueron presentados por medio de estadística descriptiva. Resultados: Obtuvo en 2015, el mayor número total de procedimientos realizados (52.801.784). Del total de procedimientos curativos entre 2015-2017, se tienen mayoría de procedimientos restauradores $(22,7 \%)$ y minoría de procedimientos quirúrgicos (15,2\%). reveló que, en los tres años estudiados, la producción de procedimientos curativos superó la de procedimientos preventivos. Conclusión: El modelo básico de atención a la salud bucal en el Nordeste presentó carácter curativo, con predominio de los procedimientos restauradores, seguidos por los periodontales, quedando en menor número los procedimientos quirúrgicos.

Descriptores: Salud Pública; Sistemas de Información en Atención Ambulatoria; Salud Bucal.

\section{INTRODUÇÃO}

De modo histórico, a prestação de serviços públicos de saúde bucal no Brasil, caracterizou-se por ações de baixa complexidade, em sua maioria curativas e mutiladoras com extrema exclusão social $^{1}$. O processo de consolidação do Sistema Único de Saúde (SUS) ampliou a finalidade das discussões sobre a organização da atenção à saúde de modo a proporcionar universalidade, abrangência de serviços, equidade, descentralização, hierarquização de serviços e controle social ${ }^{2}$.

No SUS, objetivando-se a implementação do acesso universal dos indivíduos aos serviços, a Atenção Primária à Saúde (APS) surgiu como um recurso para preconização do primeiro contato do 
usuário com o sistema de saúde por meio da Estratégia de Saúde da Família (ESF)³. A ESF vem demonstrando eficiência para reorganizar a prática na APS. É operacionalizada mediante a implantação de equipes multiprofissionais em Unidades Básicas de Saúde (UBS), responsáveis pelo acompanhamento de número definido de famílias localizadas em área geográfica adscrita ${ }^{4}$.

A inclusão de cirurgiões-dentistas na ESF foi incentivada desde o ano 2000, com a inserção da Equipes de Saúde Bucal (ESB) ${ }^{4,5}$. Essa estratégia, associada às novas Diretrizes Curriculares Nacionais (DCN) dos cursos de graduação na área de saúde, às diretrizes para a Política Nacional de Saúde Bucal (PNSB) e à Política Nacional de Atenção Básica (PNAB) contribuem para o deslocamento do campo técnico da odontologia para o campo da Saúde Bucal Coletiva (SBC) ${ }^{4,6}$.

Porém, o impulso de implantar essas estratégias com rapidez e a falta de normatização programática levou muitos gestores a incorporar ESBs pautadas na ausência de programação ${ }^{7}$. A programação de ações é, entretanto, fundamental para o diagnóstico das condições de saúde e necessidades de tratamento de uma população, permitindo estabelecer prioridades e alocar recursos de forma direcionada, por meio de práticas mais efetivas ${ }^{8}$.

A expansão da ESF trouxe a necessidade de qualificação da atenção básica ofertada à população, tornando necessário o desenvolvimento $\mathrm{e}$ implementação de ferramentas que permitissem a sua avaliação de forma permanente ${ }^{9}$. Os Sistemas de informação em saúde contêm dados populacionais e municipais úteis que auxiliam no planejamento e gestão em saúde. Sua finalidade é promover a elaboração e facilitar a avaliação de políticas, planos e programas de saúde, uma vez que seus indicadores refletem a saúde da população ${ }^{2}$.

Os únicos sistemas nacionais com registros dos procedimentos realizados pelas equipes de saúde bucal são o Sistema de Informações Ambulatoriais (SIA) e o Sistema de Informação da Atenção Básica (SIAB). Este último ainda está em desenvolvimento no que diz respeito às ações de saúde bucal ${ }^{2}$. O desafio do SUS é transformar inúmeros dados em informações úteis no retrato da saúde do país, para que o setor público possa de fato ordenar e atender às necessidades da população ${ }^{2}$.

Neste sentido, o presente trabalho tem como objetivo caracterizar o modelo de atenção básica à saúde bucal na região Nordeste do Brasil, a partir de dados secundários da produção ambulatorial em saúde bucal, disponíveis no SIA-SUS.

\section{MATERIAL E MÉTODO}

Tratou-se de estudo do tipo descritivo e quantitativo, utilizando dados secundários a partir da produção ambulatorial da atenção básica em saúde bucal (quantidade aprovada), disponíveis no Sistema de Informações Ambulatoriais do Sistema Único de Saúde (SIA-SUS).

A amostra se constituiu na produção ambulatorial em saúde bucal produzida pela região Nordeste do Brasil. Esta é uma das cinco regiões brasileiras conforme a divisão regional estabelecida pelo Instituto Brasileiro de Geografia e Estatística (IBGE). O Nordeste possui uma população estimada em 53.081.950 habitantes ${ }^{10}$. É constituída por nove estados, sendo estes: Maranhão, Piauí, Ceará, Rio Grande do Norte, Paraíba, Pernambuco, Alagoas, Sergipe e Bahia. Ocupa $18,27 \%$ do território brasileiro e possui uma área de mais de 1.500 .000 $\mathrm{km}^{2}$. A taxa de analfabetismo dessa região é de aproximadamente $18,5 \%^{10}$.

A coleta dos dados foi realizada em maio de 2018 a partir do banco de dados do Departamento de Informática do SUS (DATASUS) e considerou, para o levantamento dos procedimentos básicos em saúde bucal, o período de janeiro de 2015 a dezembro de 2017. Foram incluídos no estudo os dados sobre os procedimentos curativos e preventivos realizados em ambiente ambulatorial pelos cirurgiões-dentistas.

Os procedimentos odontológicos foram categorizados em cinco grupos para fins de avaliação, conforme a tabela de procedimentos ambulatoriais do SIA-SUS: ações preventivas [escovação dental supervisionada, aplicação tópica de flúor (coletiva e individual) e aplicação de selante (por dente)]; primeira consulta odontológica programática (referente ao primeiro exame do paciente, visando o diagnóstico e planejamento do tratamento); procedimentos restauradores (restauração em dente decíduo e permanente anterior e posterior e capeamento pulpar); procedimentos cirúrgicos (exodontia de dente decíduo e permanente, frenectomia, ulotomia e pulpotomia) e procedimentos periodontais (raspagem, alisamento, polimento supra e subgengival, raspagem corono-radicular, gengivectomia e gengivoplastia)

Após a coleta de dados, as ações preventivas e a primeira consulta odontológica programática foram categorizadas como procedimentos preventivos e os procedimentos restauradores, cirúrgicos e periodontais foram categorizados como procedimentos curativos. As informações foram coletadas por dois pesquisadores previamente treinados e registradas em formulário.

Os dados foram tabulados e analisados utilizando-se o Microsoft Excel 2016 para Windows (Microsoft Press, Redmond, WA, USA) e apresentados por meio de estatística descritiva (distribuições absolutas e percentual).

\section{RESULTADOS}

A Tabela 1 apresenta a produção ambulatorial em saúde bucal na região Nordeste do 
Brasil, no período compreendido entre 2015 a 2017, segundo os dados do SIA-SUS e organizada por categoria de procedimentos. Obteve-se em 2015, o maior número total de procedimentos realizados (52.801.784), sendo as ações preventivas os procedimentos mais executados $(31,8 \%)$. Já em 2016, realizou-se o menor número total de procedimentos entre o período avaliado (32.676.173), constituindose em maioria por ações curativas, referentes a procedimentos restauradores $(23,7 \%)$.

Do total de procedimentos curativos entre 2015-2017, tem-se maioria de procedimentos restauradores $(22,7 \%)$ e minoria de procedimentos cirúrgicos $(15,2 \%)$. Com relação ao total de procedimentos, pode-se observar que houve uma redução de 37,3\%, de 52.801 .784 em 2015 para 33.117.794 em 2017. Nota-se ainda que de 2015 a 2017, do total de 118.595 .751 atendimentos registrados, $30.713 .159 \quad(26,0 \%)$ foram ações preventivas (Tabela 1 ).

Tabela 1. Produção ambulatorial em saúde bucal, na região Nordeste, 2015-2017

\begin{tabular}{|c|c|c|c|c|c|c|c|}
\hline ANO & & $\mathbf{A P}^{*}$ & $\mathbf{P C O}^{* * *}$ & $\mathbf{P R}^{* * *}$ & $\mathbf{P C}^{* * * * *}$ & $\mathbf{P P}^{* * * * *}$ & Total \\
\hline \multirow[t]{2}{*}{2015} & $\mathrm{n}$ & 16.797 .079 & 7.540 .518 & 12.639 .367 & 6.191 .717 & 9.633 .103 & 52.801 .784 \\
\hline & $\%$ & 31,8 & 14,3 & 24,0 & 11,7 & 18,2 & 100 \\
\hline \multirow[t]{2}{*}{2016} & $\mathrm{n}$ & 7.024 .023 & 5.774 .227 & 7.759 .614 & $4.943 \cdot 388$ & 7.174 .921 & 32.676 .173 \\
\hline & $\%$ & 21,5 & 17,7 & 23,7 & 15,1 & 22,0 & 100 \\
\hline \multirow[t]{2}{*}{2017} & $\mathrm{n}$ & 6.892 .057 & 5.503 .713 & 6.509 .640 & 6.926 .554 & 7.285 .830 & 33.117 .794 \\
\hline & $\%$ & 20,8 & 16,6 & 19,7 & 20,9 & 22,0 & 100 \\
\hline \multirow[t]{2}{*}{ TOTAL } & $\mathrm{n}$ & 30.713 .159 & 18.818 .458 & 26.908 .621 & 18.061 .659 & 24.093 .854 & $118.595 \cdot 751$ \\
\hline & $\%$ & 26,0 & 15,8 & 22,7 & 15,2 & 20,3 & 100 \\
\hline \multicolumn{8}{|c|}{ onte: Ministério da Saúde/DATASUS, SIA. } \\
\hline $\begin{array}{l}* \mathrm{AP} \\
* * \mathrm{PC} \\
* * * \mathrm{P} \\
* * * * \\
* * * *\end{array}$ & $\begin{array}{l}\mathrm{R}= \\
\mathrm{PC} \\
\mathrm{PC}\end{array}$ & $\begin{array}{l}\text { es Preventi } \\
\text { rimeira Cor } \\
\text { rocediment } \\
\text { Procedimen } \\
\text { Procedime }\end{array}$ & $\begin{array}{l}\text { ilta Odontol } \\
\text { Restaurador } \\
\text { Cirúrgicos } \\
\text { s Periodont }\end{array}$ & $\begin{array}{l}\text { fica } \\
\text { es }\end{array}$ & & & \\
\hline
\end{tabular}

$\mathrm{Na}$ Tabela 2, encontram-se os dados anuais da produção ambulatorial em saúde bucal, agrupados em procedimentos preventivos e curativos. Observase que, nos três anos estudados, a produção de procedimentos curativos superou a de procedimentos preventivos. Além disso, houve um aumento no número de procedimentos curativos, de $53,9 \% \mathrm{em}$ 2015 para $62,6 \%$ em 2017 , e uma redução do número de procedimentos preventivos de 46,1\% em 2015 para $37,4 \%$ em 2017.

Tabela 2. Produção ambulatorial em saúde bucal categorizada segundo procedimentos preventivos e curativos, na região Nordeste, 2015-2017

\begin{tabular}{clcll}
\hline \multirow{2}{*}{ ANO } & \multicolumn{2}{l}{$\begin{array}{l}\text { PROCEDIMENTOS } \\
\text { PREVENTIVOS }\end{array}$} & \multicolumn{2}{l}{$\begin{array}{l}\text { PROCEDIMENTOS } \\
\text { CURATIVOS }\end{array}$} \\
\hline & $\mathrm{n}$ & $\%$ & $\mathrm{n}$ & $\%$ \\
$\mathbf{2 0 1 5}$ & 24.337 .597 & $46,1 \%$ & 28.463 .641 & $53,9 \%$ \\
$\mathbf{2 0 1 6}$ & 12.798 .250 & $39,2 \%$ & 19.877 .923 & $60,8 \%$ \\
$\mathbf{2 0 1 7}$ & 12.395 .770 & $37,4 \%$ & 20.722 .024 & $62,6 \%$ \\
\hline Fonte: Ministério da Saúde/DATASUS, SIA. & & \\
\hline
\end{tabular}

\section{DISCUSSÃO}

Os Sistemas de Informações são essenciais no sentido de construir dados para basear políticas, bem como têm sido apontados como ferramentas importantes para o diagnóstico de situações de saúde com vistas a intervenções mais aproximadas do quadro de necessidades da população ${ }^{11}$.

De acordo com o presente estudo, verificouse que houve uma redução dos procedimentos ambulatoriais, em saúde bucal, de 2015 para 2017. Para a Saúde Bucal, a questão financeira, principalmente, parece ter grande influência na sua implantação e desenvolvimento. Os gestores justificam essa atuação em função dos altos custos advindos da compra de materiais, instrumentais e equipamentos indispensáveis para o atendimento odontológico, mesmo considerando a atenção primária $^{12}$. Os dilemas referentes ao financiamento do SUS estão atrelados ao seu sub-financiamento, que é um dos pontos mais frágeis do sistema público de saúde, e que persiste como uma questão complexa e sem previsão de resolução ${ }^{13}$.

Desta forma, o desafio consiste em aumentar a coerência entre os recursos, os serviços e as necessidades da comunidade, isto é, tentar minimizar ao máximo os desequilíbrios geográficos (colocar os serviços onde estão as necessidades), numéricos (excesso de médicos, falta de enfermeiros) e organizacionais (falta de serviços básicos, abundância de serviços hospitalares) ${ }^{14}$.

Nota-se ainda que, durante o período de 2015-2017, a maioria dos procedimentos ambulatoriais realizados foram as ações preventivas. As ações de saúde bucal na ESF devem se orientar pelos princípios e diretrizes do SUS que buscam, além da ampliação do acesso da população às ações e à resolução dos problemas de saúde-doença bucal instalados, a intervenção nos fatores determinantes de saúde ${ }^{15}$.

Entretanto, analisando o total de procedimentos ambulatoriais, após categorização em preventivos e curativos, no período estudado, observou-se que os procedimentos curativos foram predominantes. No setor público, ainda persistem, em muitos municípios brasileiros, particularmente no Norte e Nordeste, sistemas de prestação de serviços odontológicos públicos pautados essencialmente na oferta de exodontias ${ }^{16}$. Esse fato também pode estar relacionado com o número reduzido de realização da primeira consulta odontológica programática, pois quanto maior a cobertura de primeira consulta odontológica programática, menor a proporção de exodontias e à medida que aumenta a razão de procedimentos coletivos, reduz a proporção de exodontias em relação ao total de procedimentos individuais $^{17}$, sendo esta uma medida preventiva de extrema importância.

Do total de procedimentos curativos entre 2015-2017, tem-se maioria de procedimentos restauradores e minoria de procedimentos cirúrgicos. Sabe-se que as principais razões que levam o 
paciente a buscar atendimento nas ESFs estão relacionadas ao alívio da dor, devolução da estética e restabelecimento da função mastigatória ${ }^{18,19}$. Por outro lado, a maioria no número de procedimentos restauradores, pode também refletir uma postura de tratamento mais conservadora e menos mutiladora ${ }^{20}$. De acordo com SB BRASIL ${ }^{21}$, em todas as faixas etárias avaliadas, o percentual de dentes com necessidade de algum tratamento para cárie é baixo. A necessidade mais frequente é de restaurações de uma superfície, sendo as regiões Norte e Nordeste as que em geral apresentam mais indivíduos com dentes que necessitam de restaurações, tratamentos pulpares ou extrações ${ }^{21}$.

No período de três anos avaliado, a produção de procedimentos curativos superou a de procedimentos preventivos. Esse fato pode estar relacionado, com a priorização da assistência à saúde bucal, durante muitas décadas, aos escolares, por meio de programas voltados para a cárie e doença periodontal, enquanto que os outros grupos populacionais acessavam aos serviços para o atendimento a situações de urgências odontológicas ${ }^{22}$.

Cardoso et al. ${ }^{23}$ corroboram com tal achado, pois observam que a atenção à saúde bucal no Brasil tem-se caracterizado pela insuficiência da oferta de procedimentos coletivos e preventivos individuais. $\mathrm{O}$ que chega a ser controverso, pois a promoção da saúde, além de ser uma das ações estratégicas da vigilância em saúde, é um dos eixos centrais estabelecidos pelo Sistema Único de Saúde ${ }^{24}$.

Para Marques e Mendes ${ }^{25}$ existem barreiras a serem rompidas para transpor o modelo de atenção tradicional, em decorrência da formação profissional tecnicista e hospitalocêntrica, pois o cirurgiãodentista e sua equipe estão basicamente envolvidos em atividades clínicas, não tendo tempo de atuar com a família, deixando de ser $\mathrm{ESF}^{25}$. Estudos conduzidos por Baldani et al. ${ }^{26}$ revelaram que, os cirurgiõesdentistas, ao serem questionados sobre os tipos de procedimentos executados pela equipe, alegaram que a falta de ações preventivas e de promoção de saúde se dá pela precariedade socioeconômica da população, visto que esses indivíduos necessitam de numerosos tratamentos, e os profissionais sentem a necessidade de adequar a oferta dos serviços à realidade local para consequente melhoria do acesso.

Diante das informações alcançadas e de sua disponibilização, os gestores públicos e as equipes de saúde não podem manter-se inertes em relação à situação atual em que se encontra a saúde bucal na região Nordeste. A possibilidade de acesso às informações precisa ser valorizada e utilizada para aprimoramento de gestão e programação de ações que melhorem a saúde da população baseando-se na sua real necessidade.

O estudo apresenta algumas limitações. Ocasionalmente, as informações podem estar comprometidas por falha de registro e/ou de controle de qualidade das informações geradas ${ }^{27}$. Normalmente são falhas relacionadas à falta de capacitação de recursos humanos, como também à falta de procura por serviços de caráter preventivo pela população ${ }^{28}$.

Porém, faz-se necessário o treinamento de profissionais da saúde para o correto preenchimento dos instrumentos para registro de dados correspondentes a saúde, sua sensibilização sobre a importância dos sistemas de informação e de como utilizar esses dados na análise e difusão das informações; e, finalmente, a capacitação dos gestores dos serviços, profissionais de saúde e usuários para a tomada de decisões ${ }^{29}$.

\section{CONCLUSÃO}

O modelo básico de atenção à saúde bucal no Nordeste apresentou caráter curativo, com predominância dos procedimentos restauradores, seguidos pelos periodontais, ficando em menor número os procedimentos cirúrgicos.

\section{REFERÊNCIAS}

1. Organização Pan-Americana da Saúde/Ministério da Saúde. Unidade Técnica de Desenvolvimento de Sistemas e Serviços de Saúde/ Coordenação Nacional de Saúde Bucal. A Política Nacional de Saúde Bucal do Brasil: registro de uma conquista histórica. (Série Técnica: Desenvolvimento de Sistemas e Serviços de Saúde) [internet]. Brasília; 2006 [acesso em 30 junho 2018]. Disponível em: http://189.28.128.100/dab/docs/publicacoes/geral/ serie_tecnica_11_port.pdf

2. Linhares LL, Bordin R. Oral healthcare profile of a Brazilian state capital according to data from the Outpatient Clinic Information Database of the Unified National Health System. Rev Gaúcha Odontol. 2012; 60(1):41-7.

3. Solano MP, Bulgarelli PT, dos Santos CM, Mestriner SF, Mestriner Jr W, Mesquita LC et al. Utilização de serviços de saúde bucal na atenção primária: perspectivas dos usuários do sus de um município do sul do Brasil. Ciência Plural. 2017; 3(3):81-92.

4. Reis WG, Scherer MDA, Carcereri DL. O trabalho do Cirurgião-Dentista na Atenção Primária à Saúde: entre o prescrito e o real. Saúde debate. 2015; 39(104):56-64.

5. Brasil. Ministério da Saúde. Portaria $n^{\circ} 2.488$, de 21 de outubro de 2011. Aprova a Política Nacional de Atenção Básica, estabelecendo a revisão de diretrizes e normas para a organização da Atenção Básica, para a Estratégia Saúde da Família (ESF) e o Programa de Agentes Comunitários de Saúde (Pacs). Diário Oficial da República Federativa do Brasil [internet]. Brasília; 2011. [acesso em 30 junho 2018]. Disponível em: http://bvsms.saude.gov.br/bvs/saudelegis/gm/2011 /prt2488_21_10_2011.html 
6. Botazzo C, Chaves SCL. Saúde Bucal Coletiva: Antecedentes e estado da arte. In: Botazzo, C. Diálogos sobre a boca. São Paulo: Hucitec; 2013.

7. Zanetti CHG. Por um caminho sustentável para universalização da atenção básica: saúde bucal da família com equidade e integralidade. Brasília: Pólo UnB/Planaltina SUS-DF de Ensino e Pesquisa em Saúde Bucal-Departamento de Odontologia da Universidade de Brasília, 2000.

8. Mota E, Carvalho DM. Sistemas de Informação em Saúde. In: Rouquaryol MZ, Almeida Filho N. Epidemiologia e Saúde. 5.ed. Rio de Janeiro: Medsi; 1999. p.505-521.

9. Brandão ALRB, Giovanella L, Campos CEA. Avaliação da atenção básica pela perspectiva dos usuários: adaptação do instrumento EUROPEP para grandes centros urbanos brasileiros. Ciênc. saúde coletiva. 2013;18(1):103-14.

10.Brasil. Ministério do Planejamento Orçamento e Gestão, Brasil. Instituto Brasileiro de Geografia e Estatística. Censo demográfico 2010. Instituto Brasileiro de Geografia e Estatística [internet]. 2010 [acesso em 30 junho 2018]. Disponível em: http://www.ibge.gov.br.

11. Medeiros KR, Machado HOP, Albuquerque PC, Gurgel Junior GD. O Sistema de Informação em Saúde como instrumento da política de recursos humanos: um mecanismo importante na detecção das necessidades da força de trabalho para o SUS. Cienc saude coletiva. 2005; 10(2):433-40.

12. Mattos GCM, Ferreira EF, Leite ICG, Greco RM. A inclusão da equipe de saúde bucal na Estratégia Saúde da Família: entraves, avanços e desafios. Ciênc. saúde coletiva. 2014; 19(2):373-82.

13.Souza VPR, Muniz STG. Dilemas do financimento do sistema único de saúde (SUS): um estudo dos recursos aplicados pela união, estado do paraná e municípios da região metropolitana de Curitiba $-2^{\circ}$ Seminário Nacional de Planejamento e Desenvolvimento Universidade do Estado de Santa Catarina (UDESC), Florianópolis, SC. 2014.

14.Dussault G. A gestão dos serviços públicos de saúde: características e exigências. Rev Adm Publica. 1992; 26(2):8-19.

15. Andrade KLC, Ferreira EF. Avaliação da inserção da odontologia no Programa Saúde da Família de Pompeu (MG): a satisfação do usuário. Cienc saude coletiva. 2006; 11(1):123-30.

16. Roncalli AGCO. Levantamentos Epidemiológicos em Saúde Bucal no Brasil. In: Antunes JLF, Peres MA. Epidemiologia da Saúde Bucal. Rio de Janeiro: Guanabara Koogan; 2006. p.32-48.

17. Brasil. Ministério da Saúde. Portaria n ${ }^{\circ} 493$, de 10 de março de 2006. Aprova a Relação de Indicadores da Atenção Básica - 2006, cujos indicadores deverão ser pactuados entre municípios, estados e Ministério da Saúde. Brasilia, 2006.
18. Halling A, Ordell S. Emergency dental service is still neededalso for regular attenders within a comprehensive insurance system. Swed Dent J. 2000; 24(5-6):173-81.

19. Mialhe FL, Possobon RF, Boligon F, Menezes MA. Medo odontológico entre pacientes atendidos em um serviço de urgência. Pesq Bras Odontoped Clin Integr. 2010; 10(3):483-87.

20.Fonseca DAV, Mialhe FL, Ambrosano GMB, Pereira AC, Meneghim MC. Influência da organização da atenção básica e das características sociodemográficas da população na demanda pelo pronto atendimento odontológico municipal. Ciênc. saúde coletiva. 2014; 19(1):269-78.

21.Brasil. Ministério da Saúde. Secretaria de Atenção à Saúde. Secretaria de Vigilância em Saúde. SB Brasil 2010: Pesquisa Nacional de Saúde Bucal: Resultados Principais [Internet]. Brasília; 2011 [cited 2017 March 05]. Available from: http://bvsms.saude.gov.br/bvs/publicacoes/pesquis a_nacional_saude_bucal.pdf

22.Brasil. Ministério da Saúde. Conselho Federal de Odontologia. II Conferência Nacional de Saúde Bucal - Relatório Final. Divulgação do Comitê Executivo da II CNBS. Brasília, 1993. 28p.

23. Cardoso ACC, Santos Jr. RQ, Souza LEP, Barbosa MBCB. Inserção da equipe de saúde bucal no PSF um desafio para melhoria da qualidade de atenção à saúde. Rev baiana saúde pública. 2002; 26(1/2):94-8.

24.Kusma SZ, Moysés ST, Moysés SJ. Promoção da saúde: perspectivas avaliativas para a saúde bucal na atenção primária em saúde. Cad Saúde Pública. 2012; 28(Suppl):s9-19.

25. Marques RM, Mendes A. A política de incentivos do Ministério da Saúde para atenção básica: uma ameaça à autonomia dos gestores municipais e ao princípio da integralidade? Cad Saude Pública. 2002; 18(Supl.):163-71.

26.Baldani MH, Fadel CB, Possamai T, Queiroz MGS. A inclusão da odontologia no Programa de Saúde da Família no estado do Paraná, Brasil. Cad Saúde Publica. 2005; 21(4):1026-35.

27. Teixeira ND, Facchini LA, Castilho ED. Avaliação da evolução da demanda de saúde bucal através do uso de sistemas de informação em saúde. Rev enferm saúde. 2011; 1(1):50-9.

28. Silva LS, Santana KR, Pinheiro HHC, Nascimento LS. Indicadores de atenção básica e especializada em saúde bucal nos municípios do Estado do Pará, Brasil: estudo ecológico, 20012010. Epidemiol Serv Saúde. 2013; 22(2):325-34.

29.Barros SG, Chaves SCL. A utilização do Sistema de Informações Ambulatoriais (SIA-SUS) como instrumento para caracterização das ações de saúde bucal. Epidemiol Serv Saúde. 2003; 12(1):41-51. 


\section{CONFLITO DE INTERESSES}

Os autores declaram não haver conflitos de interesse.

AUTOR PARA CORRESPONDENCIA

Isla Camilla Carvalho Laureano

carvalhoisla@gmail.com

Submetido em 03/07/2018

Aceito em 04/10/2018 